\title{
Onset of Mechanical Stability in Random Packings of Frictional Spheres
}

\author{
Melissa Jerkins, Matthias Schröter, ${ }^{*}$ and Harry L. Swinney ${ }^{\dagger}$ \\ Center for Nonlinear Dynamics and Department of Physics, University of Texas at Austin, Austin, Texas 78712, USA \\ Tim J. Senden, Mohammad Saadatfar, and Tomaso Aste \\ Department of Applied Mathematics, Research School of Physical Sciences and Engineering, The Australian National University, \\ 0200 Canberra, ACT, Australia \\ (Received 15 November 2007; revised manuscript received 28 April 2008; published 2 July 2008)
}

\begin{abstract}
Using sedimentation to obtain precisely controlled packings of noncohesive spheres, we find that the volume fraction $\phi_{\mathrm{RLP}}$ of the loosest mechanically stable packing is in an operational sense well defined by a limit process. This random loose packing volume fraction decreases with decreasing pressure $p$ and increasing interparticle friction coefficient $\mu$. Using $\mathrm{x}$-ray tomography to correct for a container boundary effect that depends on particle size, we find for rough particles in the limit $p \rightarrow 0$ a new lower bound, $\phi_{\mathrm{RLP}}=0.550 \pm 0.001$.

DOI: 10.1103/PhysRevLett.101.018301

PACS numbers: 45.70.Cc, 46.65.+g, 47.57.ef
\end{abstract}

Introduction. - If granular materials such as sand, sugar, or snow are excited strongly (e.g., by shaking or shearing), they exhibit fluidlike behavior. However, after the excitation stops, dissipation quickly produces a static packing that is mechanically stable under its own weight. Experiments [1-8] and simulations [9-13] have shown that the volume fraction has a well-defined lower limit, $\phi_{\mathrm{RLP}}$, called random loose packing (RLP).

The value of $\phi_{\text {RLP }}$ depends on the particle-particle interactions. Packings of cohesive particles like fine powders are stable under their own weight for values of $\phi_{\text {RLP }}$ as low as 0.15 [5-8]. However, many granular materials do not exhibit cohesive forces. Simulations of frictionless elastic noncohesive spheres have found the onset of a finite bulk modulus at the jamming point, $\phi_{J} \approx 0.64$ [9-11]. Real spheres have friction and then it has been suggested that $\phi_{\text {RLP }}$ depends on the density difference between the particles and the surrounding fluid [1-4]. The lowest volume fraction reported thus far, $\phi_{\mathrm{RLP}}=0.555$, was observed for slowly sedimenting spheres in a liquid of nearly the same density [3].

Here we demonstrate a limit process that yields welldefined values of $\phi_{\text {RLP }}$ that depend on pressure and coefficient of friction. The results are discussed in the context of a statistical mechanics approach based on the ensemble of all mechanically stable configurations [14].

Experiment.-Mechanically stable packings of glass spheres were prepared by allowing the particles to sediment following flow pulses in a water fluidized bed. The fluidized bed was contained in a vertical polycarbonate tube with an inner diameter $D$ of $12.8 \mathrm{~mm}$ and a length of $230 \mathrm{~mm}$. The tube's bottom end was closed by a distributor consisting of a porous bronze disk (height, $8 \mathrm{~mm}$; nominal pore size, $25 \mu \mathrm{m}$ ). A programmable syringe pump (Harvard Apparatus) created pulses of constant flow rate $Q$. During a flow pulse of 2 min length the granular medium fluidized and expanded until it reached a steady state height. After each flow pulse, the particles sedimented onto the distributor and formed a mechanically stable packing whose volume fraction depended on $Q$, as shown in Fig. 1. A higher value of $Q$ resulted in a more expanded fluidized bed, longer sedimentation time, and lower $\phi$ of the sediment. Packings created in this way are independent of the state of the sample prior to the last flow pulse [15]. This property is important for any statistical mechanics approach [16]. The volume fractions in Fig. 1 are averaged over the whole sample: $\phi=$ $m / \rho A h_{\text {sed }}$, where $\rho$ is the particle density, $m$ is the total mass of all the spheres, $A$ is the cross sectional area of the tube, and $h_{\text {sed }}$ is the height of the sedimented sample determined from images.

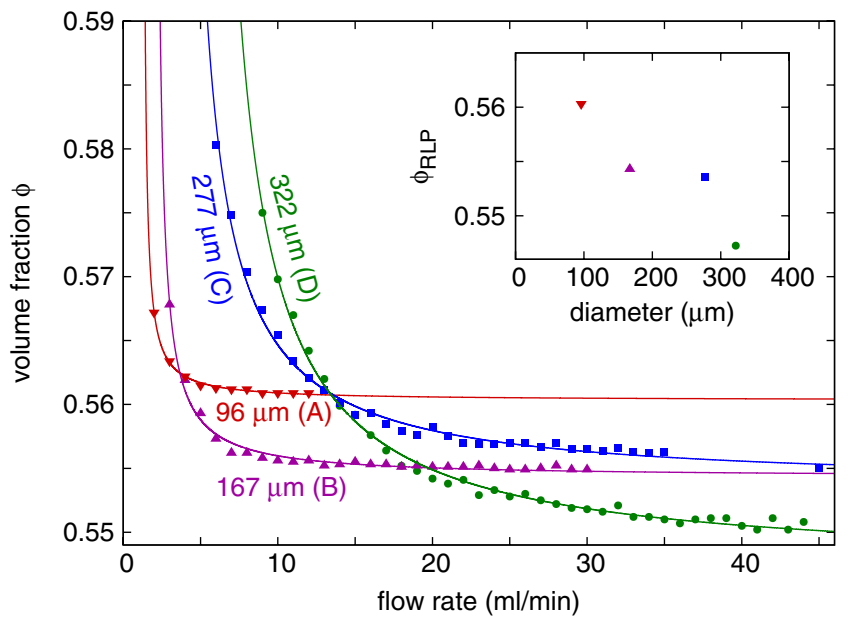

FIG. 1 (color online). The approach to random loose packing in a limit process is achieved using flow pulses in a liquid fluidized bed. Data for different particle diameters were fit to (1) to obtain $\phi_{\text {RLP. }}$. Letters in parentheses refer to the particle samples in Table I. Inset: Diameter dependence of $\phi_{\text {RLP }}$ without container size correction (see text). Sample height was $97 \mathrm{~mm}$ at RLP; five flow pulses were averaged for each flow rate. 
TABLE I. Properties of the different samples of glass spheres. Particle diameters $d$ and standard deviations $\sigma$ were measured with a Camsizer (Retsch Technology). Angles of repose under water were averaged over 10 measurements.

\begin{tabular}{ccrcc}
\hline \hline Sample & $d(\mu \mathrm{m})$ & $\sigma(\%)$ & Supplier & Angle of repose \\
\hline$A$ & 96 & 15.6 & Cataphote & $24.8 \pm 1.0$ \\
$B$ & 167 & 16.1 & Cataphote & $26.1 \pm 0.7$ \\
$C$ & 277 & 7.6 & Cataphote & $25.3 \pm 0.8$ \\
$D$ & 322 & 9.3 & Cataphote & $25.5 \pm 0.7$ \\
$E$ & 261 & 5.0 & MoSci & $24.0 \pm 0.8$ \\
$F$ & 257 & 7.8 & Cataphote & $27.7 \pm 1.3$ \\
$G$ & 255 & 2.7 & MoSci & $26.6 \pm 0.7$ \\
\hline \hline
\end{tabular}

The properties of the different samples of particles are given in Table I. The density $\rho$ of the particles in each sample was measured with an accuracy of $0.06 \%$ using a Gay-Lussac specific gravity bottle and a Micromeritics AccuPyc 1330 gas pycnometer; the average $\rho$ was $2.48 \mathrm{~g} / \mathrm{cm}^{3}$. To characterize the frictional properties of the samples we measured the angle of repose under water: a beaker containing a layer of particles about $5 \mathrm{~mm}$ high was tilted until the particles started to move. To obtain an especially rough sample $(F)$ we soaked part of sample $C$ for $3 \mathrm{~h}$ in hydrofluoric acid. Sample $E$ consists of spheres that were smoothed by exposure to more than 45000 flow pulses in a fluidized bed [15].

RLP is defined by a limit process. - The main improvement over earlier studies using sedimenting particles [3,7] is that our control of $Q$ allows us to change the sedimentation time independent of the liquid density. This procedure reveals the convergence of $\phi$ to $\phi_{\text {RLP. }}$ The observation that the slowest relaxing preparation yields the loosest packings agrees with simulations of frictional disks and spheres [11,12]. Figure 1 shows that $\phi(Q)$ is well described by the fit function used in [15],

$$
\phi(Q)=\phi_{\mathrm{RLP}}+\frac{a}{Q-b},
$$

which we use to determine $\phi_{\mathrm{RLP}}$.

Dependence on particle diameter.-The inset of Fig. 1 indicates that $\phi_{\mathrm{RLP}}$ decreases with particle diameter; however, this decrease is due to lower volume fraction near a container wall, an effect known since the earliest studies [1]. This effect is explained in Fig. 2(a): since particles cannot penetrate the container wall, voids are larger there and the volume fraction of the layer adjacent to the boundary is lower than $\phi_{\text {bulk }}$ measured in the core of the sample. The difference between $\phi_{\text {apparent }}$ averaged over the whole container and $\phi_{\text {bulk }}$ increases with the ratio $d / D$ and produces the trend displayed in the inset of Fig. 1.

We examined the finite size effect using $x$-ray tomography [17], Fig. 2. In each run positions of $1.5 \times 10^{5}$ spheres were measured with a resolution of better than $0.1 \%$ of a sphere diameter [18]. Figure 2(c) shows the difference between the apparent volume fraction using all particles and the bulk volume fraction (measured using the Voronoi volumes [18] of all particles that are at least $4 d$ away from the container walls). For $Q<10 \mathrm{ml} / \mathrm{min}$, $\phi_{\text {apparent }}$ values from the tomographic measurements agree with the results (dotted curve) for the $277 \mu \mathrm{m}$ particles in Fig. 1, but for $Q>10 \mathrm{ml} / \mathrm{min}$ the tomographic values are larger due to unavoidable vibrations during the recording of the tomogram.

The inset of Fig. 2(c) shows the difference between the bulk and apparent $\phi$ as a function of $\phi_{\text {apparent }}$. A linear fit yields
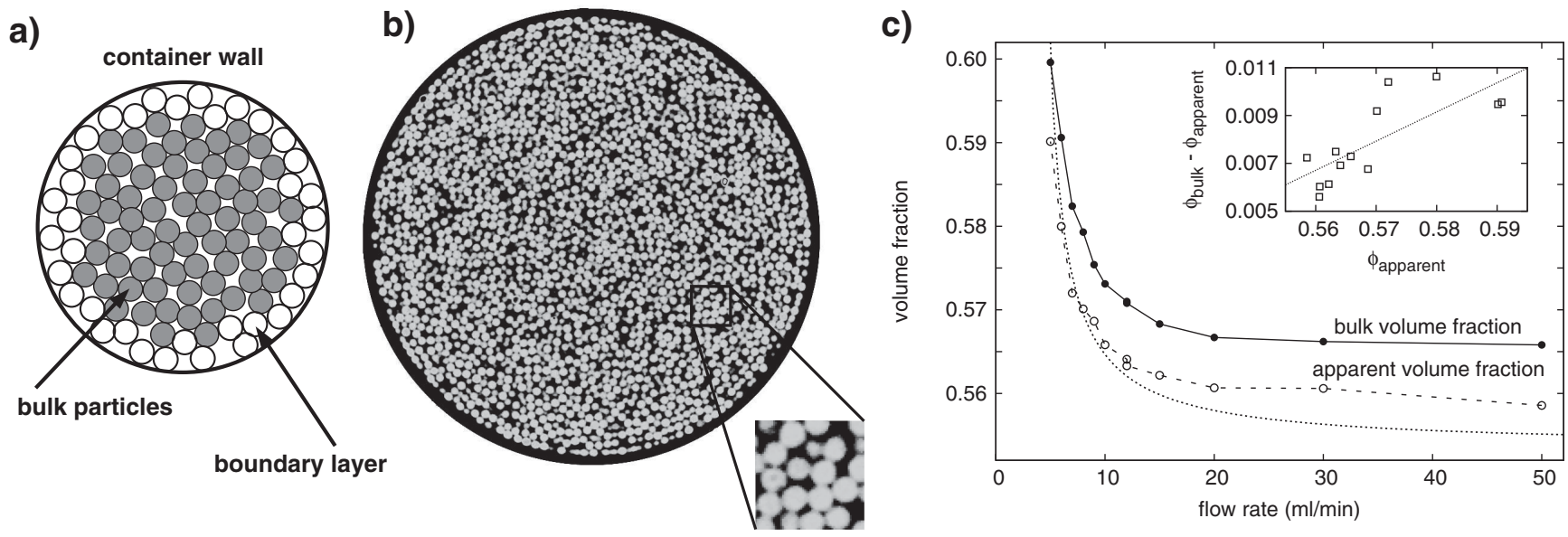

FIG. 2. (a) Illustration of the necessity of a finite size correction of the volume fraction determined from the total sample volume. Particles near the boundary have a lower volume fraction than those in the bulk. (b) Cross section of a 3-dimensional x-ray tomogram of the fluidized bed; the particles have a diameter of $255 \mu \mathrm{m}$ (sample $G$ ). The inner diameter of the polycarbonate tube (black circle) is $12.8 \mathrm{~mm}$. (c) Bulk volume fraction for particles that are at least $4 d$ away from boundary, and the apparent volume fraction $\phi_{\text {apparent }}$ for all particles. The dotted line corresponds to the fit to the $277 \mu \mathrm{m}$ particles in Fig. 1. The inset shows a linear fit (2) to the difference between bulk and apparent volume fractions. Sample height was $39 \mathrm{~mm}$ at RLP. 


$$
\phi_{\text {bulk }}=\phi_{\text {apparent }}+0.122\left(\phi_{\text {apparent }}-0.505\right) .
$$

For all further experiments we used only spheres with diameter 261 or $257 \mu \mathrm{m}$ (sample $E$ or $F$ ), and we corrected for the effect of finite container size using (2).

Influence of pressure. - The stress inside a column of grains differs from the hydrostatic case in two ways: (i) anisotropy - the horizontal stress $\sigma_{x x}$ in the column differs from the vertical stress $\sigma_{z z}$; (ii) wall friction-the part of the load carried by the frictional sidewalls increases with depth $z$ below the surface. Consequently, $\sigma_{x x}$ and $\sigma_{z z}$ saturate with $z$. In our analysis we use a pressure dependence on height given by the Janssen model [19], which assumes a constant stress ratio $K=\sigma_{x x} / \sigma_{z z}$ everywhere in a sample. Experiments show that this model is a fair approximation in the absence of external loads [20,21]. The model gives a saturation of pressure $\left(p=\sigma_{z z}\right)$ with depth,

$$
p(z)=p_{\text {sat }}\left(1-e^{-z / l}\right)
$$

to a constant value $p_{\text {sat }}=\Delta \rho g D / 4 K \mu_{W}$, where $\mu_{W}$ is the coefficient of particle-wall friction, $g$ is the gravitational acceleration, $\Delta \rho$ is the density difference between spheres and surrounding liquid, and $l=D / 4 K \mu_{W}$. Equation (3) indicates two ways of controlling the pressure distribution inside the column: (I) Increasing the sample height, which increases the fraction of the sample at $p_{\text {sat }}$. If $\phi_{\text {RLP }}$ increases with $p$, then the average $\phi_{\text {RLP }}$ measured by our method should increase with sample height. This behavior is confirmed in Fig. 3. (II) Decreasing the density difference $\Delta \rho$, which decreases $p_{\text {sat }}$ but keeps the pressure profile unchanged. We increased the fluid density to as high as $2.39 \mathrm{~g} / \mathrm{cm}^{3}$, close to the $2.48 \mathrm{~g} / \mathrm{cm}^{3}$ particle density, by adding sodium polytungstate to the water. Results

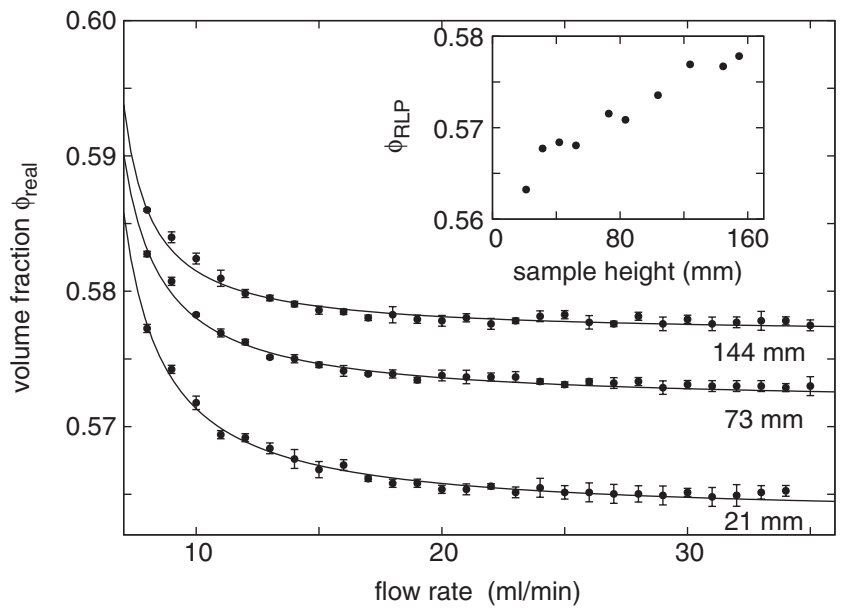

FIG. 3. Dependence of $\phi$ on $Q$ for different sample heights (261 $\mu \mathrm{m}$ spheres, sample $E$ ). Values of $\phi$ were corrected for finite size effect using (2). Solid lines are fits to (1). Inset: The resultant $\phi_{\text {RLP }}$ values as a function of sample height. for different $\Delta \rho$ (Fig. 4) again confirm that $\phi_{\text {RLP }}$ decreases with decreasing $p$.

A limit $\phi_{\mathrm{RLP}}^{0}$ would be given by matching the fluid and particle densities, but in this limit there would be no sedimentation and no connected granular packings would form. Therefore, we extrapolate to determine $\phi_{\mathrm{RLP}}^{0}$ : In the absence of theory we follow [5] and use the pressure dependence close to the jamming point known for frictionless static soft spheres [9-11,22] and frictionless thermal hard spheres [23]:

$$
\phi_{\mathrm{RLP}}=\phi_{\mathrm{RLP}}^{0}+\left(\frac{\Delta \rho}{a}\right)^{\alpha},
$$

where we identify $\Delta \rho \sim p_{\text {sat }}$. A fit of $\phi_{\text {RLP }}$ for smooth particles (sample $E$ ) in Fig. 4 yields $\phi_{\mathrm{RLP}}^{0}=0.555 \pm$ 0.006 . The value of $\alpha=0.51 \pm 0.25$ is approximate because our derivation of (4) did not take into account the $\phi$ dependence of $K$ [20].

Influence of frictional properties. - Figure 4 shows that $\phi_{\text {RLP }}$ for the rough spheres was lower than for the smooth spheres. For the rough spheres a fit to (4) yields $\phi_{\mathrm{RLP}}^{0}=$ $0.550 \pm 0.001$ and $\alpha=0.89 \pm 0.16$. The decrease of $\phi_{\mathrm{RLP}}$ and $\phi_{\mathrm{RLP}}^{0}$ with increasing friction agrees with another experiment [24], model [25], and simulations [11-13,26].

Discussion. - Our experimental results and numerical simulations [11,12] both show that RLP is well defined in an operational sense: in the limit of infinitesimally slow preparation, the volume fraction of a sample converges to $\phi_{\text {RLP }}$, independent of the details of preparation. Care should be taken in comparing theory for frictionless hard spheres with the experimental results, in part because of the different possible ways of defining mechanical stability $[27,28]$.

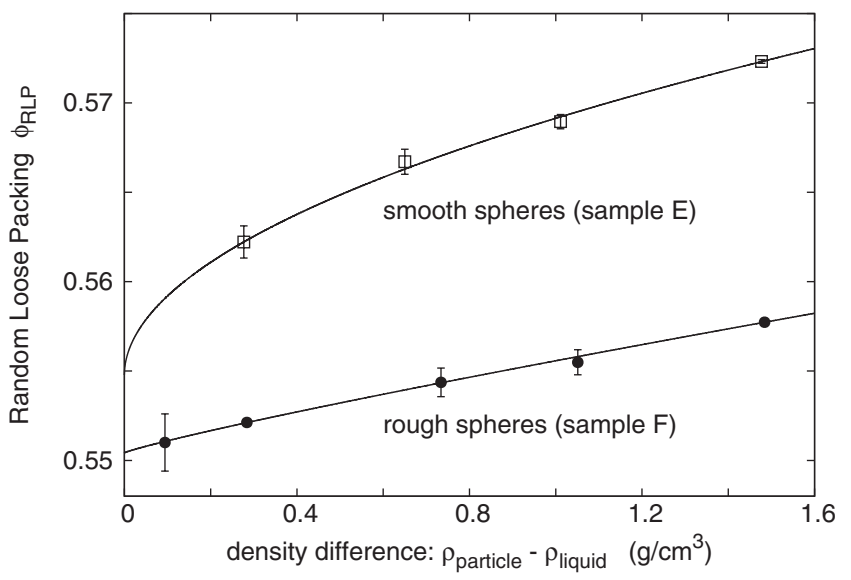

FIG. 4. $\phi_{\text {RLP }}$ as a function of the density difference between particles and fluid, for smooth $(261 \mu \mathrm{m})$ and rough $(257 \mu \mathrm{m})$ particles. Solid lines are fits to (4). Data have been corrected for the finite size effect using (2). Sample height was $64 \mathrm{~mm}$, and the points are averages obtained at the highest possible flow rate, since for small density differences the flow rate range was too small for a meaningful fit with (1). 
The observation of a well-defined $\phi_{\text {RLP }}$ can be considered within the framework of a statistical mechanics of static granular material [14], where a configurational entropy $S$ is defined as the logarithm of the number of mechanically stable configurations for a given $\phi, p$, and friction coefficient. Two different approaches can explain RLP using two different assumptions of how $S$ depends on $\phi$. The first approach assumes that RLP is the smallest $\phi$ where $S$ becomes larger than zero. This is compatible with the existence of looser, highly ordered configurations [27], as their number seems not to grow exponentially with system size, so $S=0$.

The second approach is supported by numerical results on the number of stable configurations of frictional disks [26], where $S$ has a maximum at RLP. This idea agrees with slow sedimentation leading to RLP; it is simply the most probable configuration. If the sedimentation speed is increased, the additional kinetic energy allows the system to explore the local energy landscape and find rarer but lower potential energy (denser) configurations. Further, the maximum of $S$ and therefore RLP moves to higher values of $\phi$ for decreasing friction [26]. This agrees with our results and with simulations of frictionless disks that have a maximum of $S$ at random close packing [29]. Our results indicate also that increasing $p$ shifts the maximum of $S$ in a similar way.

Conclusions. - Mechanically stable packings of spheres prepared with increasing sedimentation time display a lower bound of their volume fraction, $\phi_{\mathrm{RLP}}$, which depends on the pressure and the coefficient of friction but not on the diameter of the spheres. In the limit of zero pressure we have found a new lowest value of $\phi_{\text {RLP }}$, $0.550 \pm 0.001$.

We thank Massimo Pica Ciamarra and Antonio Coniglio for sharing their unpublished results, and Brandon McElroy for his assistance with the Camsizer. Further we thank W. D. McCormick, Narayanan Menon, Charles Radin, Leo Silbert, Jack Swift, and participants of the Aspen Jamming workshop for helpful discussions. This work was supported by Robert A. Welch Foundation Grant No. F-0805.

*matthias.schroeter@ds.mpg.de

†swinney@chaos.utexas.edu

[1] G. Scott, Nature (London) 188, 908 (1960).

[2] R. Rutgers, Nature (London) 193, 465 (1962).

[3] G. Y. Onoda and E. G. Liniger, Phys. Rev. Lett. 64, 2727 (1990).
[4] R. Ojha, N. Menon, and D. J. Durian, Phys. Rev. E 62, 4442 (2000).

[5] J. M. Valverde, M. A. S. Quintanilla, and A. Castellanos, Phys. Rev. Lett. 92, 258303 (2004).

[6] J. M. Valverde and A. Castellanos, Europhys. Lett. 75, 985 (2006).

[7] K. J. Dong, R. Y. Yang, R. P. Zou, and A. B. Yu, Phys. Rev. Lett. 96, 145505 (2006).

[8] P. B. Umbanhowar and D. I. Goldman, Am. J. Phys. 74, 720 (2006).

[9] H. A. Makse, D. L. Johnson, and L. M. Schwartz, Phys. Rev. Lett. 84, 4160 (2000).

[10] C. S. O'Hern, S. A. Langer, A. J. Liu, and S. R. Nagel, Phys. Rev. Lett. 88, 075507 (2002); C. S. O'Hern, L. E. Silbert, A. J. Liu, and S. R. Nagel, Phys. Rev. E 68, 011306 (2003).

[11] H.P. Zhang and H. A. Makse, Phys. Rev. E 72, 011301 (2005).

[12] K. Shundyak, M. van Hecke, and W. van Saarloos, Phys. Rev. E 75, 010301(R) (2007).

[13] Leonardo Silbert (private communication).

[14] S. Edwards and R. Blumenfeld, in Granular Physics, edited by A. Mehta (Cambridge University Press, Cambridge, England, 2007).

[15] M. Schröter, D. I. Goldman, and H. L. Swinney, Phys. Rev. E 71, 030301(R) (2005).

[16] T. Aste and T. Di Matteo, Phys. Rev. E 77, 021309 (2008).

[17] A. Sakellariou, T. J. Sawkins, T. J. Senden, and A. Limaye, Physica (Amsterdam) 339A, 152 (2004); T. Aste, M. Saadatfar, A. Sakellariou, and T.J. Senden, Physica (Amsterdam) 339A, 16 (2004); T. Aste, M. Saadatfar, and T. J. Senden, Phys. Rev. E 71, 061302 (2005).

[18] T. Aste, T. D. Matteo, M. Saadatfar, T. J. Senden, M. Schröter, and H. L. Swinney, Europhys. Lett. 79, 24003 (2007).

[19] M. Sperl, Granular Matter 8, 59 (2006).

[20] L. Vanel and E. Clément, Eur. Phys. J. B 11, 525 (1999).

[21] L. Vanel, P. Claudin, J.-P. Bouchaud, M.E. Cates, E. Clément, and J.P. Wittmer, Phys. Rev. Lett. 84, 1439 (2000).

[22] T. S. Majmudar, M. Sperl, S. Luding, and R. P. Behringer, Phys. Rev. Lett. 98, 058001 (2007).

[23] C. Brito and M. Wyart, Europhys. Lett. 76, 149 (2006).

[24] Narayanan Menon (private communication).

[25] Y. Srebro and D. Levine, Phys. Rev. E 68, 061301 (2003).

[26] Massimo Pica Ciamarra and Antonio Coniglio, arXiv:0805.0220.

[27] S. Torquato and F.H. Stillinger, J. Appl. Phys. 102, 093511 (2007).

[28] S. Torquato and F. H. Stillinger, J. Phys. Chem. B 105, 11849 (2001)

[29] G.-J. Gao, J. Bławzdziewicz, and C. S. O’Hern, Phys. Rev. E 74, 061304 (2006). 